\title{
We should reframe the discussion/debate about neonatal repair of tetralogy of Fallot
}

\author{
Charles D. Fraser, Jr, MD
}

Feature Editor's Introduction-It is my honor to introduce the expert opinions of Drs Jonas and Fraser, 2 highly gifted and respected surgeons, whom the congenital editors invited to share their not always congruent expert opinions on the surgical management of neonates with tetralogy of Fallot (ToF). Isn't this a "dead horse" issue that had already been pulverized to ash? In a certain sense, yes, but a prestigious cardiology journal recently published a report based on national administrative data that concluded "complete surgical repair for neonates with ToF is associated with a significantly higher risk for early and 2-year mortality compared with the staged approach, after accounting for patient and hospital characteristics." " Given what we know about administrative databases, we would likely need to invoke a bit of faith regarding the validity of this specific conclusion. But what was shocking was that the best 2-year survival was only 85\%. Really? This is ToF-low single-digit mortality, right? Apparently wrong! This sobering, humbling fact should cause us significant pause. Apparently there is a much bigger horse afoot very much alive and well. So I encourage you to pause, clear your thoughts (and biases), and proceed to enjoy our experts' opinions.

\section{Ronald K. Woods, MD, PhD}

I am grateful for the opportunity to weigh in, once again, on the subject of the surgical treatment of tetralogy of Fallot (ToF). The invitation, as I understand it, is for me to provide an "Expert Opinion" arguing against a policy of routine neonatal complete repair of ToF. I expect that at many levels, the readership of the Journal has grown weary of debates about ToF repair; in fact, as I verbally contemplated my commentary, I found myself asking, "Aren't people tired of hearing about tetralogy?" My hope is that we all

From the Texas Center for Pediatric and Congenital Heart Disease, Dell Children's Medical Center, Surgery and Pediatrics, University of Texas, Dell Medical School, Austin, Tex.

Received for publication April 7, 2020; revisions received May 29, 2020; accepted for publication May 30, 2020; available ahead of print June 10, 2020.

Address for reprints: Charles D. Fraser, Jr, MD, Dell Children's Medical Center, 4900 Mueller Blvd, Suite 3S.003, Austin, TX 78723 (E-mail: Charles.fraser@austin. utexas.edu).

J Thorac Cardiovasc Surg 2021;161:1421-5 0022-5223/ $\$ 36.00$

Copyright (c) 2020 Published by Elsevier Inc. on behalf of The American Association for Thoracic Surgery

https://doi.org/10.1016/j.jtcvs.2020.05.093

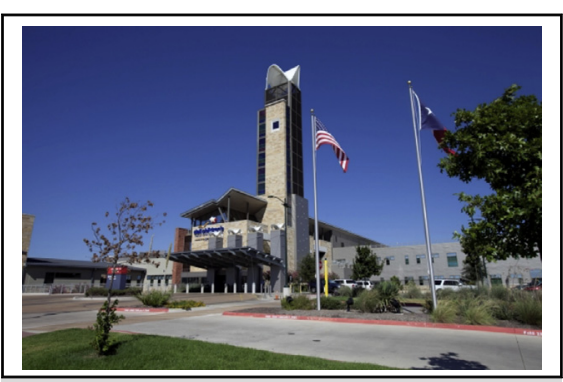

At Dell Children's, we favor an individualized approach to ToF repair.

CENTRAL MESSAGE

We need to reframe our focus on the surgical treatment of ToF toward a more holistic, individualized strategy measured by meaningful longitudinal outcomes.

This Invited Expert Opinion provides a perspec tive on the following paper: J Am Coll Cardiol. 2019;74(12):1570-1579. https://doi.org/10.1016/j.jacc 2019.05.057.

realize that there is much work to do on this subject, as recent evidence suggests.

While I risk sounding like a politician, I believe that the debate should be reframed or redefined. I argue that the core questions for ToF repair should be not only when we should perform a complete repair but also how? A derivative of that theme is the question of whether there is a place for palliation in the treatment algorithm? A core issue that we must agree on is that to understand performance in the treatment of ToF, we must change to longitudinal outcomes measures beyond the historical frame of reference, 30-day, or inhospital mortality rates. I believe my counterpart in this "debate" agrees with this notion.

To start this dialogue, I believe we must come to a common understanding that surgically repaired ToF is a chronic disease state. As such, in making my points about the 
timing and strategy for treatment, I believe we now have, more than ever before, the opportunity to begin with the end in mind in ToF treatment.

\section{SURGICALLY REPAIRED TETRALOGY OF \\ FALLOT: A CHRONIC DISEASE STATE}

Essentially, no patient who has undergone ToF repair is "cured." Volumes have been written about the late sequelae of pulmonary insufficiency, tricuspid regurgitation, residual atrial and ventricular level shunting, dysrhythmias (atrial and ventricular), and distorted branch pulmonary arteries. We do not know the true prevalence of these problems in repaired $\mathrm{ToF}$ because the late follow-up series largely comprise single-institution, cohort series. Furthermore, we do not really know what matters most to patients with repaired ToF over the course of their lifetimes. It is true we know some things, but longitudinal, patient-derived outcomes measures are almost completely lacking. Thus, my first argument about ToF treatment is that we need better outcomes measures for patients with repaired ToF. These should include patient-derived outcomes measures. Patients with ToF should be followed from the time of diagnosis for the rest of their lives with objective, best available outcomes measures. We definitely have the technology and ability to do these things. Only by becoming more granular in our assessment of outcomes will we be able to continue to refine our treatment methodologies.

\section{THIRTY-DAY AND IN-HOSPITAL MORTALITY RATES FOR TETRALOGY OF FALLOT TREATMENT ARE INADEQUATE TO CLARIFY OPTIMUM TREATMENT STRATEGIES}

During previous debates, my counterparts have argued that some centers, through nefarious intent to inflate their operative mortality statistics, have deferred primary, complete corrections of ToF (and other lesions) to defer risk and at some levels double count operations. This is, of course, quite a distasteful and somewhat offensive accusation. Without doubt, performing an interval newborn palliation for a patient with ToF followed by infant complete repair represents 2 operations, ideally in the first year of life. One question to ask in counter-argument is whether it is then better to perform a newborn ToF repair (more to come on the differences in the operations later) followed by a higher incidence of remedial surgery in infancy (which is well documented)? These pro/con positions become unsolvable with current outcomes methodologies; 30-day mortality rates are far too crude to resolve this disagreement. Thus, I believe one core point we should all be able to agree on is that the management of ToF should be judged on an intention to-treat basis starting at the time of diagnosis. Furthermore, as per the previous section, we should be assessing outcomes over the long term. The 30-day window of follow-up is easily achievable, but completely inadequate. Based on new methodologic changes from the Society of Thoracic Surgeons Congenital Heart Disease database, participating centers will be required to provide 1 -year follow-up data on indexed surgical operations. This is a good start, but there is still much to be learned beyond 1 year of follow-up, and as noted, I believe it would be most useful to have patient outcomes organized by disease category rather than by operation. Surely, we all agree that what a parent wants to know, among many things, is the likelihood that their child will be alive and functionally well at 1 year and beyond, irrespective of treatment strategy debate.

In the context of this, the article "2-Year Outcomes After Complete or Staged Procedure for Tetralogy of Fallot in Neonates," published in the Journal of the American College of Cardiology in 2019 by a group of investigators from Children's Hospital of Philadelphia (CHOP), provides fascinating substrate for further discussion and is the provoking publication for this invited commentary. ${ }^{1}$ My role is not to critique this article, but several points merit attention. The study is derived from a large, administrative dataset (Pediatric Health Information System database), and as with all administrative database studies, there is room for criticism of the data. Having said that, the overall data bring a concerning signal. Whether the authors have demonstrated, as the article claims, that complete neonatal ToF repair is more risky than staged repair can be debated (the study observed $84 \%$ 2-year survival for staged repair vs $77 \%$ for primary neonatal repair). What cannot be debated, however, is that both strategies are associated with more significant 2-year attrition than contemporary practice acknowledges. In my experience, most of us quote a much lower operative mortality rate for complete ToF repair (irrespective of age) or palliation. We have published on this issue, and it is routine for me to quote a 30-day mortality risk estimate for ToF repair of less than $1 \% .^{2}$ Am I undervaluing the ongoing morbidity associated with ToF surgery? Probably so, and I argue that most institutions probably are too. We are fixated on the acute event and, thus far, very incomplete in long-term analysis.

\section{TETRALOGY OF FALLOT PRESENTING WITH SYMPTOMATOLOGY IN THE NEONATAL PERIOD REPRESENTS A DISEASE SPECTRUM}

In the current era, many (perhaps most) babies diagnosed with ToF are discovered during routine fetal ultrasound screening. Most are asymptomatic and minimally desaturated during the true neonatal period (emphasis is that we are talking about ToF with pulmonary stenosis, not pulmonary atresia). It is curious, therefore, to observe that a number of centers have promoted a policy of routine, complete neonatal $\mathrm{ToF}$ repair, irrespective of symptoms. Why is this? Several theories have been promulgated. One is that prograde right ventricular outflow tract flow optimizes pulmonary vasculature 
growth (including microvasculature)-completely unproven. Another is that it is best for the developing brain in newborns with ToF to be exposed to normal or nearnormal oxygen delivery to optimize brain development-also unproven and in no way objectively studied longitudinally (more to come on that issue). It has always seemed very curious to me that centers that strongly advocate neonatal ToF repair on the basis of theoretical brain protection will expose babies to lengthy periods of profound hypothermia and total circulatory arrest to execute the operation-this seems counterintuitive. On the other hand, a legitimate argument for neonatal repair is to minimize the risk of unheralded hypercyanosis. This is yet another argument for the intention-to-treat analysis paradigm suggested previously.

There is an ugly side of this dilemma: inter-institutional competition. I have had a number of surgical colleagues tell me over the course of the last 25 years that in their region, they are forced (usually by the referring cardiologists) to proceed with neonatal ToF repair or risk losing the patient referral to a competing institution. This is an unfortunate reality that must be called into question. It cannot be in the patient's best interest to offer any operation based on a desire to keep the patient in one's institution. This happens, and it shouldn't. It certainly is not a legitimate argument for neonatal ToF repair.

Not all patients with ToF are created equal. As per above, most will be asymptomatic in the newborn period. Others present with overt cyanosis, complex anatomy, and multiple associated comorbidities. Genetic abnormalities are categorically known to add risk to surgical repair in most lesions. To suggest a "one size fits all" or uniform neonatal reparative strategy for all newborns irrespective of the details of their presenting status undervalues the complexities of perioperative outcomes. It seems more logical to me to individualize the treatment strategy based on the uniqueness of each individual. For some, that may include the benefit of an interval staging palliation to optimize the patient's condition at the time of complete repair. Perhaps this is part of the "signal" of the article by Savia and colleagues. ${ }^{1}$

For symptomatic newborns with ToF, it is not clear that outcomes are uniformly optimized by a neonatal complete repair as has been strongly promoted by some institutions. As per the article, ${ }^{1}$ there appears to be some legitimate reason to challenge a uniform strategy to repair all newborns. Perhaps in response to their own article, other investigators from $\mathrm{CHOP}$ have more recently published a follow-up article on their own experience. ${ }^{3}$ As they note in their article, "the optimal management of tetralogy of Fallot in symptomatic neonates remains unknown." It must be emphasized that these are data from an outstanding center that has prided itself on being an epicenter for newborn cardiac surgery. Important additional data the CHOP group has provided in this series encompassing patients undergoing either primary neonatal repair or palliation and subsequent repair at CHOP between 2000 and 2013 include the following:

1. There is significant mortality for neonatal primary repair (7/112 patients).

2. A large number of newborns undergoing complete repair are exposed to deep hypothermic circulatory repair.

3. Patients undergoing complete neonatal repair had more postprocedure cardiac events compared with patients undergoing staged repair.

4. Early morbidity was greater in patients undergoing neonatal complete repair.

5. Overall, there was comparable 2-year survival in comparing primary with staged strategy, but there were a number of patients who were lost to follow-up.

All food for thought.

\section{NEWBORN BRAINS ARE VULNERABLE}

As previously noted, one of the arguments that has been historically used for routine neonatal ToF repair is to "protect the brain" through improved oxygen delivery. Palliation is criticized from many perspectives, including lack of normal oxygenation and the realities of ongoing intracardiac mixing (and thereby embolic potential). I have already made mention of the inconsistencies in this argument from centers that also use circulatory arrest. Furthermore, it is widely accepted, in fact it is taught as a surgical principle, that if one is to optimize survival in neonatal ToF repair, it is desirable, perhaps mandatory, to leave an atrial level communication to mitigate the effects of acute right heart failure. This also countermands the argument against palliation; patients after neonatal repair are routinely left with the potential (reality) of right to left atrial level shunting, intracardiac mixing, and potentially desaturation.

In the sphere of brain development and neuroprotection, I argue that we truly do not know which strategy is optimal. We do know a lot more now than ever about neurodevelopmental risk. We know that newborns with congenital heart disease have immature brains relative to their agematched peers with normal hearts. ${ }^{4}$ We know that if we look carefully, we can find evidence of brain injury in the majority of newborns requiring cardiac surgery before their operations (particularly if we look at magnetic resonance imaging evidence of brain injury before surgery). We have evidence that the methodology of cardiopulmonary bypass may affect neurodevelopment potential. ${ }^{5}$ What we do not know is the best strategy for ToF repair in terms of neurodevelopmental outcomes. One can envision rational arguments against an aggressive complete repair strategy in a fragile newborn. What is best for the preterm or small for gestational age baby with comorbidities that might include intraventricular hemorrhage, necrotizing enterocolitis, premature lung disease, or other common problems? 
Is such a patient's brain better protected by a period on cardiopulmonary bypass with profound systemic anticoagulation, with or without circulatory arrest, or a less-invasive palliation that may offer the potential of subsequent repair under less risky circumstances? We simply do not know and will not know without the sort of robust longitudinal outcomes measurements as recommended previously.

\section{ARE ALL PALLIATIONS BAD?}

Some have argued that any sort of palliative approach for ToF is outdated and not consistent with the advances that have been made in neonatal cardiac surgery for other conditions. This is a curious argument in an era when very complex single ventricle conditions are and must be palliated to achieve longitudinal success. My view is again that the issue should be reframed. Surely, we agree that stage I palliation for hypoplastic left heart syndrome is necessary and the only pathway to success for this condition. Why do we impugn palliation in other circumstances where there may be some real benefit to the patient for deferred complete repair? As in the circumstance described, if the risk of complete repair in a given individual is suboptimal, an interval palliation of a less-invasive nature may offer an idealized opportunity for success. Is this the signal we are seeing in the article by Savia and colleagues?

Moreover, it has been argued that palliations carry a higher overall risk, including higher risk of distorted branch pulmonary arteries. No doubt, a poorly constructed Blalock-Taussig-Thomas shunt or improperly positioned ductal stent may result in badly distorted pulmonary arteries or excessive pulmonary blood flow-these palliations must be constructed accurately and judiciously. To argue, however, that it is easier to perform an accurate, delicate, and durable neonatal complete correction is to me quite counterintuitive. An accurate operation is an accurate operation whether it is a palliative operation or a complete correction. The goal must be optimization.

\section{ARE ALL "COMPLETE" REPAIRS EQUIVALENT?}

In debating strategies for complete ToF repair, I believe it is important to emphasize that many of us do a very different operation for definitive repair. In my opinion, this must influence the timing of the operation. This is a concept that should be familiar and logical to most congenital heart surgeons, for example, that there is an optimal timeframe for a definitive operation in a given individual. This should not be a conceptual problem for us; for example, we know that timing of repair of complete atrioventricular septal defect is usually more ideal beyond the neonatal period to optimize repair of the atrioventricular valves. Shouldn't it be logical that there an ideal time for an individual's ToF repair?

Another curiosity of the debate is that, depending on where one was trained or relative to institutional policy, we surgeons perform complete ToF repair in very different ways. The classically taught technique includes a significant infundibulotomy with or without continuation through the pulmonary valve annulus: the transventricular approach. This right ventricle (RV) incision affords direct access to the ventricular septal defect for closure and allows direct relief of the right ventricular outflow tract obstruction. It is an easy operation and is the most frequently practiced operation for ToF repair, but it comes at a long-term cost. My opinion is that the price is measured in suboptimal outcomes that become apparent over the life of the patient, not usually in terms of acute outcomes. The key is that this operative technique sacrifices a contractile infundibulum. In many patients, this RV incision also involves the transection of major infundibular coronary arteries. There is ample evidence that the infundibulum is important to overall RV function, and this is the primary criticism I have about this approach; there are significant long-term consequences to this operation.

The alternative to the transventricular operation is to avoid a significant transmural infundibulotomy; the transatrial-transpulmonary (TA/TP) approach as originally described by Kawashima ${ }^{6}$ and largely championed by Karl and colleagues, ${ }^{7}$ and subsequent secondgeneration congenital heart surgeons. The primary premises of this approach include complete avoidance of a ventriculotomy and preservation of all major epicardial coronary arteries. The ventricular septal defect is closed by a transatrial approach, and all infundibular resection is performed from within, through the tricuspid valve, pulmonary valve, or both. I believe this is a superior operation if one considers the long-term optimization. It is important to emphasize, however, that the TA/TP approach is more difficult, particularly in smaller hearts. This is where the debate about surgical timing becomes nuanced, but important. The TA/TP operation is possible in many newborns with ToF, but not uniformly so. Thus, those of us who are strict proponents of this methodology favor non-neonatal, infant repair to optimize the operation.

\section{AN IDEALIZED TETRALOGY OF FALLOT REPARATIVE STRATEGY}

In my opinion, the following represents an idealized reparative strategy for ToF:

1. No attrition while awaiting repair and avoidance of hypercyanosis

2. Optimized branch pulmonary artery architecture and growth

3. Optimized early and late RV function through avoidance of transmural RV incision

4. Optimized pulmonary valve function

5. Avoidance of tricuspid valve distortion 
6. Avoidance of early (junctional ectopic tachycardia, atrioventricular block) and late dysrhythmias (right bundle branch block, atrial tachycardia/fibrillation, ventricular tachycardias)

7. Avoidance of reparative techniques that subject the patient to deep hypothermic circulatory arrest

8. Optimized neurodevelopmental potential

9. Individualized management based on unique patient characteristics at the time of presentation strategically coordinated to optimize the preceding elements, including the judicious use of palliation when necessary

10. $100 \%$ patient follow-up (for life)

Arguably, if these elements can be satisfied with a newborn repair, it is justifiable. If not, a safe interim palliation that stages the patient to an infant repair is a superior strategy.

\section{CONCLUSIONS}

The "debate" regarding the surgical treatment of ToF needs to be updated. I think the issue is not neonatal versus non-neonatal or complete repair versus interim palliation. The true question is given the large experience we have with ToF care and the many tools we now have to offer, can we optimize early and lifelong outcomes in these patients through individualized strategies that take advantage of creative solutions, including judicious palliations, optimized repairs, and holistic follow-up? Current evidence would suggest we have significant room for improvement.

\section{Conflict of Interest Statement}

The author reported no conflicts of interest.

The Journal policy requires editors and reviewers to disclose conflicts of interest and to decline handling or reviewing manuscripts for which they may have a conflict of interest. The editors and reviewers of this article have no conflicts of interest.

\section{References}

1. Savia JJ, Faerber JA, Huang YV, Zaoutis T, Goldmuntz E, Kawut SM, et al. 2-year outcomes after complete or staged procedure for tetralogy of Fallot in neonates. $J$ Am Coll Cardiol. 2019;74:1570-9.

2. Morales DL, Zafar F, Heinle JS, Ocampo EC, Kim JJ, Relyea K, et al. Right ventricular infundibulum sparing (RVIS) tetralogy of Fallot repair: a review of over 300 patients. Ann Surg. 2009;250:611-7.

3. Bailey J, Elci OU, Mascio CE, Mercer-Rosa L, Goldmuntz E. Staged versus complete repair in the symptomatic neonate with tetralogy of Fallot. Ann Thorac Surg. 2020;109:802-9.

4. Licht D, Shera DM, Clancy R, Wernovsky G, Montenegro LM, Nicolson SC, et al Brain maturation is delayed in infants with congenital heart defects. J Thorac Cardiovasc Surg. 2009;137:529-37.

5. Andropoulos DB, Easley RB, Brady K, McKenzie DE, Heinle JS, Dickerson HA et al. Changing expectations for neurologic outcomes after the neonatal arterial switch operation. Ann Thorac Surg. 2012;94:1250-6.

6. Kawashima Y, Kitamura S, Nakano S, Yagihara T. Corrective surgery for tetralogy of Fallot without or with minimal right ventriculotomy and with repair of the pulmonary valve. Circulation. 1981;64(pt 2):145-53.

7. Karl TR, Sano S, Pornviliwan S, Mee RBB. Tetralogy of Fallot: favorable outcome of nonneonatal transatrial, transpulmonary repair. Ann Thorac Surg. 1992;54: 903-7. 